Procrastinación y factores relacionados para su análisis en la educación superior.

Procrastination and related factors for analysis in higher education.

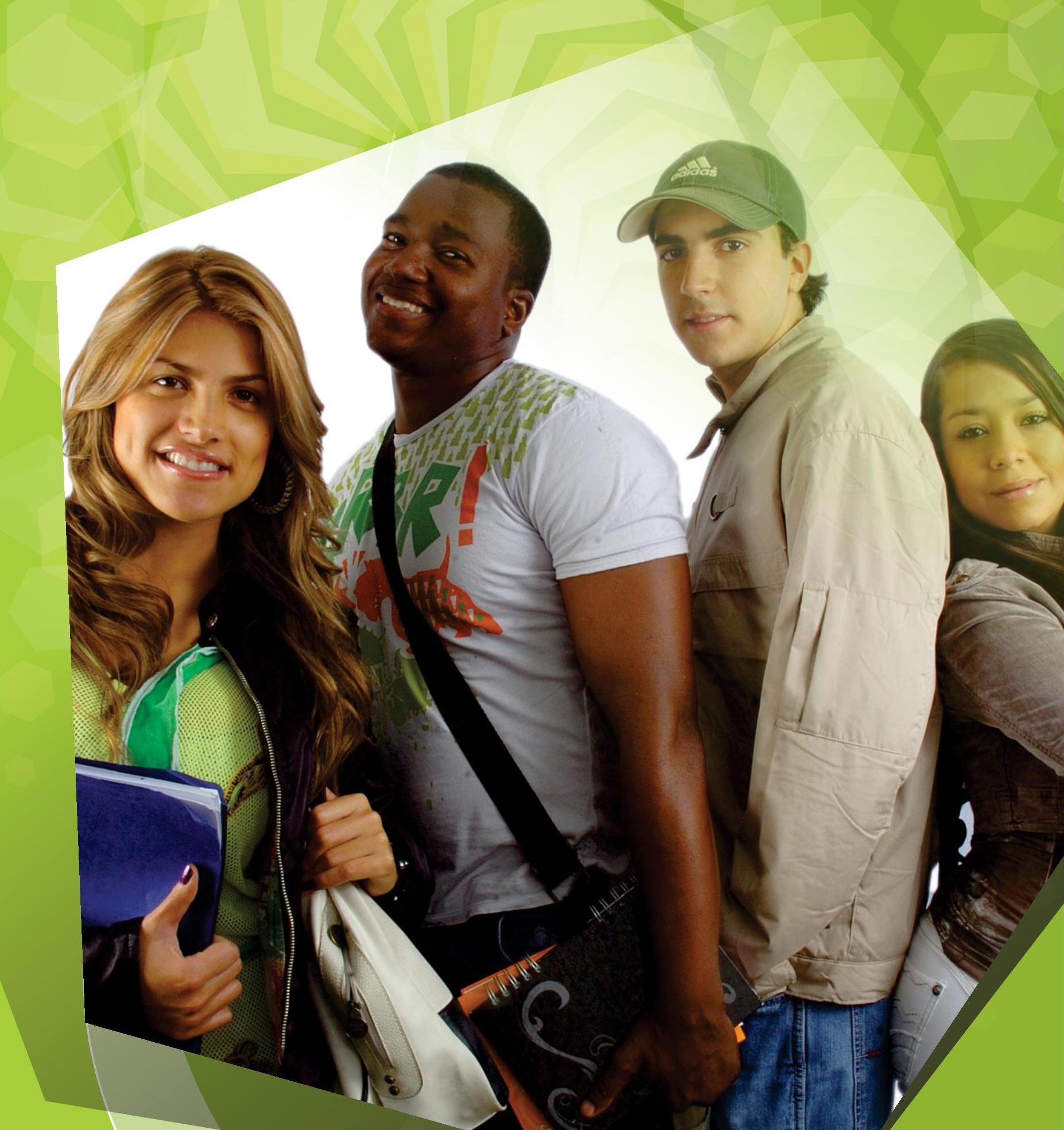




\title{
Procrastinación y factores relacionados para su análisis en la educación superior. ${ }^{1}$ Procrastination and related factors for analysis in higher education.
}

\author{
Carlos Alberto Gómez Cano ${ }^{2}$, Natalia Fernanda Ortiz Palomá ${ }^{3}$, Lina María \\ Perdomo Rojas ${ }^{4}$ \\ Universidad de la Amazonia, Caquetá, Florencia, Colombia.
}

Artículo recibido en Marzo de 2016; artículo aceptado en mes Mayo de 2016

Citación del artículo: Gómez C, CA., Ortiz, N. \& Perdomo, L. (2106). Procrastinación y factores relacionados en la educación superior. I+D Revista de Investigaciones, 7(1), 32-39.

\begin{abstract}
Resumen
La procrastinación o permanencia irregular es una de las problemáticas que afecta a los estudiantes de pregrado, a las universidades y a la comunidad, puesto que si un estudiante permanece en su carrera más del tiempo estimado incurrirá en mayores esfuerzos individuales e institucionales. Por lo tanto, en aras de determinarlos factores a los que obedece la permanencia irregular, se realizó una investigación descriptiva y cualitativa, mediante el análisis documental de los antecedentes investigativos relacionados con la procrastinación, con el fin de plantear estrategias que contrarresten esta problemática que afecta la culminación de estudios universitarios.
\end{abstract}

Palabras clave: Procrastinación, Permanencia irregular, Retención, Deserción.

\footnotetext{
Abstract

Procrastination or irregular permanence, is one of the problems affecting undergraduates, universities, and community, because if a student remains in his career over estimated time incur greater individual and institutional efforts. In this regard, in order to determine the factors that obeys the irregular stay a descriptive, qualitative research was conducted by the documentary analysis of the research background related to procrastination, therefore, they were identified as investigated nationally and international,
}

factors to consider in higher education, in order to devise strategies to counteract this problem that affects the culmination of college.

Keywords: Procrastination, Irregular permanence, Retention, Desertion.

\section{Introducción}

Los estudios sobre la afectación de las condiciones académicas en la educación superior son muy comunes en nuestros tiempos; existe gran variedad de problemas, perspectivas de análisis y formas de interpretación. Para el caso específico de la permanencia académica, los estudios abordan desde la vinculación y motivación de los programas académicos hasta la estrecha relación entre estudiante-currículo-docente; además, existen posiciones académicas en torno a los factores endógenos y exógenos que tienen incidencia sobre la vida universitaria.

En este artículo, la permanencia académica será abordada desde la dimensión del tiempo, es decir, la relación existente entre el tiempo establecido en el plan de estudios y el trasegar académico de los estudiantes, el cual, en condiciones de normalidad debe ser exactamente el mismo (Gómez \& Tovar, 2016). Así las cosas, cuando exista diferencia entre el tiempo y el recorrido académico del estudiante, se tipifica permanencia irregular.

\footnotetext{
${ }^{1}$ Artículo de revisión documental y descriptivo, con enfoque cualitativo. Universidad de la Amazonia de la ciudad de Florencia, Caquetá (Colombia).Campus Porvenir Calle 17 Diagonal 17 con Carrera 3F - Barrio Porvenir, Tel: (+57) 84340591.

${ }^{2}$ Contador Público, Administrador Público, Esp. en Pedagogía y en Gestión Pública, Est. Maestría en Ciencias de la Educación. Docente del Programa de Contaduría Pública de la Universidad de la Amazonia. Grupo de Estudio de Futuro en el Mundo Amazónico (GEMA). Correo electrónico: carlosgomez325@gmail.com

${ }^{3}$ Estudiante de pregrado de Administración de empresas, Facultad de Ciencias Contables, Económicas y Administrativas, Universidad de la Amazonia, de la ciudad de Florencia, Caquetá (Colombia). Correo electrónico:nortiz384@gmail.com

${ }^{4}$ Estudiante de pregrado de Administración de empresas, Facultad de Ciencias Contables, Económicas y Administrativas, Universidad de la Amazoniade la ciudad de Florencia, Caquetá (Colombia). Correo electrónico: linaperdomo26@hotmail.com
} 
Sobre el particular,Regueyra(2014) expone que la procrastinación académica o permanencia irregular, es considerada como la acción de retrasar actividades que deben atenderse, sustituyéndolas por otrasirrelevantes o agradables, como los momentos de ocio y diversión, que no guardan relación con el recorrido académico.

La procrastinación académica es concebida como un comportamiento disfuncional, en el que se excusan o justifican retrasos y se evita la culpa cuando el sujeto se encuentra frente a una tarea académica. Sánchez(2010),buscó realizar una aproximación a los principales factores relacionados con este fenómeno, encontrando variables endógenas y exógenas que inciden en el desarrollo del estudiante dentro de su formación académica.

Los factores que influyen en la permanencia irregular de los estudiantes universitarios se convierten en una hoja de ruta y análisis al interior de lasentidades de educación superior, a fin de generar los mecanismos de intervención necesarios en cada institución; en tal sentido, en este estudio se determinan y describen algunos de ellos.

De acuerdo con lo mencionado, en el presente artículo se describe primero, cómo se desarrolló este estudio descriptivo, mediante la revisión documental de tesis, artículos y ponencias publicadas en la web sobre los factores relacionados con la procrastinación; segundo, los referentes teóricos que pueden ser retomados en estudios relacionados con el tema de la presente indagación y, finalmente, se presenta cada uno de los factores y aspectos que pueden ser retomados en lasuniversidadespara identificar problemáticas relacionadas con la permanencia irregular y generar alternativas para el fomento de la graduación de los estudiantes de pregrado del sistema de educación superior.

\section{Método}

Para el desarrollo del presente artículo se aborda un enfoque cualitativocon el método documental y descriptivo. En el quese consultaron diferentes documentos, como tesis, artículos científicos y estudios investigativos en bases de datos (Gómez et al, 2016); y posteriormente, se describió lo más importante.

De acuerdo con lo mencionado, la investigación se realizó en las siguientes fases:

- Exploratoria: Se consultaron documentos de carácter académico y científico publicados entre el 2010 al 2015, los cuales se condensaron en una matriz plana de resultados.

- Clasificación y sistematización: con la información obtenida, se clasificaron los autores y referentes, ordenados y condensados según sus posiciones y temáticas abordadas.

- Estructuración: Finalmente, se hilaron los postulados y resultados de la búsqueda para la consolidación de los resultados, logrando generar un balance y discusión de la cuestión.

\section{Fuentes y procedencia de la información.}

Los estudios encontrados de acuerdo a su orden en los años son: $2010(36 \%), 2012(16 \%), 2013(16 \%), 2014$ (16\%), $2011(12 \%)$, y 2015 (4\%); respecto a la procedencia de los documentos, se hallóque los autores son principalmente originarios en su orden, de España, Argentina, Chile, México, Perú, Costa Rica, Estados Unidos, Venezuela, Nicaragua, Uruguay, Suecia, Romania, con una participación del $64 \%$, el restante (36\%) son de origen Colombiano.

\section{Resultados y discusión \\ Ámbito internacional, procrastinación, dilación o permanencia irregular en el pregrado.}

Al revisar la literatura en el ámbito internacional es abundante la información que se registra sobre el fenómeno de procrastinación. Por ejemplo, González (2010) propone que la motivación y actitud del estudiante son factores clave para el éxito de la formación universitaria; en la misma línea, Rizo (2010) sostiene que las mayores dificultades en los universitarios radica en el desarrollo de trabajos finales, la presión por los compromisos financieros adquiridos para poder acceder a la educación y la motivación hacia el porvenir;Tonconi (2010), sostiene que la permanencia irregular tiene estrecha relación con la cantidad de créditos matriculados; Cuchacovich (2011)plantea que las universidades tienen grandes deficiencias debido a la ausencia de orientación profesional que permita un verdadero acercamiento a las necesidades y aspiraciones de los estudiantes, situación que es validada por Carbajal (2012)y García (2014), quienes exponen que el abandono se produce especialmente durante el primer año, lo cual es un problema no solo para el joven, sino para la universidad y para la sociedad, generando saturación de los escenarios y programas académicos (Escanés et al, 2013); además, Peyet al (2013) sostienen que se debe evaluar la duración de los procesos de formación, sugiriendo una reducción de los tiempos en los planes de estudio.

Carrión et al (2012) exponen que la ausencia de rigurosos sistemas de formación en los niveles de media y básica, generan trastornos en el desarrollo académico de los estudiantes universitarios. En un nivel más profundo, Hirschhorn (2010) expone la importancia de flexibilizar y dinamizar la elaboración de tesis, como una forma de reducir los obstáculos existentes, logrando disminuir la procrastinación sobre el tema. Esta afirmación es validada por Rodríguez et al (2012), quienes concluyen que los estudiantes están dispuestos a desarrollar sus trabajos de grado, pero carecen de atención oportuna por parte de las universidades.

Sobre los planos personales de los educandos, Regueyra (2014) establece que los vínculos laborales también 
influyen en la dispersión sobre los estudiantes, pues su tiempo se limita, generando desatención con los compromisos académicos; Prieto \& Raleigh (2013)sostienen que estos escenarios generan conductas de ansiedad que conducen a la postergación de tareas por la presencia del miedo al fracaso.

En términos de prevención, Escanéset al (2013), sostienen que es imperioso que cada universidad genere sistemas de información y detección de la procrastinación, generando de esta manera intervención oportuna que disminuya el riesgo de abandono. En este sentido, Natividad (2014), sostiene que la procrastinación es un fenómeno poco analizado, lo que genera un esfuerzo de cada universidad por dar solución a sus incógnitas. En palabras de Buentello et al (2014) y Trejo \& Sierra (2014), es necesario crear redes de talentos, identificando un tipo de política de diáspora para reducir la disminución en la formación académica y la perdida de capital humano.

Finalmente, Ulloa et al (2015) concluyen que el principal detonante del abandono es la diferencia entre las expectativas personales y las metas institucionales, situación que sepuede atender de manera oportuna en cada institución, logrando disminuir los índices de procrastinación.

\section{Ámbito nacional, procrastinación, dilación o permanencia irregular en el pregrado.}

Para el caso de Colombia, tal y como se detalló en la metodología, se consultaron varios autores relacionados con el fenómeno de procrastinación, encontrando hallandomúltiples aristas de análisis e interpretación. Por ejemplo, Calvo (2014) expone que los bajos niveles de control, disfrazados de movilidad académica, pueden generar permanencia irregular en los sistemas universitarios. Desde otra posición, Ramírez \&Vélez (2010) enfatizan en la importancia de identificar los tipos de deserción académica,según las condiciones de cada institución para generar alternativas de intervención mitigar los impactos causados por los fenómenos inherentes al abandono escolar; situación que es todo un desafío para los claustros educativos (Torres, 2010).Sin embargo, esta tarea en Colombia es maratónica, pues no se cuentan con estudios que caractericen el problema de la procrastinación académica en la universidad (Sánchez, 2010).

Ahora bien, sobre el caso motivacional, Zarate \&Socha (2010), expone que se debe empoderar a los estudiantes universitarios de su rol, pues en los procesos de transición académica se generan niveles de irresponsabilidad frente a los procesos educativos, que generaran, en el futuro, mayores costos y desgaste institucional.Lo anterior, según Universidad Distrital Francisco José de Caldas (2011), también tiene incidencia enel prestigio institucional ya que la eficiencia institucional es medida por la dinámica de la graduación.
Como posibles mecanismos de intervención, se han generado algunas propuestas en el seno de las universidades, por ejemplo, Molina \& Contreras (2012)plantean la necesidad de crear programas de apoyo financiero, que garanticen la permanencia. Estaposición es compartida por Lopera \& Ortiz (2012), quienes además proponen la generación de apoyos académicos e investigativos. Para Torres (2012), todos estos elementos deben ser contemplados en un plan de retención institucional.

En tal sentido, la Universidad de Antioquia, a través del proyecto ALFA (2013), ha iniciado una serie de acciones que propenden por identificar aquellos factores que inciden negativamente en el estudiante y que lo llevan a la deserción o el fracaso universitario; estos esfuerzos, según Pineda (2013), además de su impacto institucional, han generado una serie de proposiciones que, además de generar datos de interés,aportan al cumplimiento de las obligaciones y estándares de las autoridades competentes en materia educativa del país (Villa et al, 2013).

Respecto a los esfuerzos para mitigar el fenómeno existen varias iniciativas al interior de las Universidades, las cuales poseen diferentes métodos y estructuras, justificadas en el uso de la autonomía universitaria para atender contextos específicos. Por ejemplo, Barragán et al (2010)sostienen que la heterogeneidad de los estudiantes que ingresan al sistema educativo debe ser objeto de intervención temprana, situación que para Parra \& Rodríguez (2014) y Peralta \& Mora (2014), debe ser atendida a través de estrategias de permanencia universitaria, que correspondan a escenarios específicos y concretos, generando verdaderos momentos de intervención.

\section{Factores relacionados con la procrastinación}

Retención: La retención se refiere a la capacidad institucional para mantener vinculado a un estudiante en riesgo de deserción, desde su admisión hasta su graduación (Suarez \& Díaz, 2015). En consecuencia, según Donoso et al (2010) las estrategias de retención más exitosas están ligadas a políticas de intervención flexibles y contextualizadas que atiendan realmente los escenarios institucionales. La retención, según Pineda et al (2011), debe estar caracterizada desde una mirada institucional, por la generación de motivaciones solidas en los estudiantes.

Finalmente, Montes \& Lerner (2011) plantean la importancia de observar las variables de retención desde una perspectiva integral, no solo bajo el reflejo de lo cualitativo, sino dando paso también a lo cuantitativo, permitiendo generar verdaderos escenarios de análisis, reflexión e interacción (Caballero et al, 2012)

Deserción: Entendida de forma plana como el abandono de los estudios, situación que según Gómez (2010)puede generarse por motivaciones externas (del ser) e internas (institucionales), que inciden en el trasegar académico. 
Para Olivete (2010), el estrés académico es una de las causas endógenas más fuertes de deserción, por lo que se requiere un plan de transformación de estos contextos, a fin de impedir el abandono; pues, según, De Vries et al (2010), la decepción en los estudiantes dilata los riesgos de deserción.

Para la atención de este fenómeno, Mori (2012) concluye que es necesario contar con espacios de interacción fuera de aula que permitan generar reflexiones y motivaciones para la permanencia, además de canalizar espacios de orientación vocacional, apoyos económicosacadémicos y procesos de integración a la vida universitaria, los cuales facilitarán la estancia en el sistema educativo (Castañeda, 2013; Dupéré et al, 2015)

Finalmente, según Freeman\&Simonsen (2015), los procesos de investigación sobre la deserción son imperiosos, pues de estos se sustraen insumos sólidos para la toma de decisiones y una oportuna intervención de los agentes involucrados (Shefi, 2015; Frelin, 2015).

Modelo pedagógico y enfoques de aprendizaje:Sobre el particular, Arreola (2012) planteó la importancia de apoderar a los integrantes de la comunidad académica sobre los modelos pedagógicos, a fin de incentivar su participación como factor de éxito institucional.

Ahora bien, para el enfoque de competencias,García (2010) deja en evidencia la carencia de modelos y orientaciones para integrar la evaluación por competencias en los sistemas educativos, dejando en entre dicho uno de los retos de la educación en nuestros tiempos (Hernández, 2010; Vallejos, 2012).

Lo anterior, obliga a una reflexión profunda en torno los modelos y enfoques institucionales, pues estos deben ser aliados del proceso, lo que supone intervención de sus agentes de manera sinérgica, con el único objetivo de convertirlo en un aliado institucional.

\section{Conclusiones}

La procrastinación académica es un fenómeno que demanda total atención de las universidades, ya que su proliferación genera impactos en varios escenarios y dimensiones. Por ejemplo, desde una posición humana, implica insatisfacción por no alcanzar el desarrollo esperado, generando consigo frustración para los educandos; y desde una posición institucional, genera traumatismos relacionados con cobertura, disponibilidad y exceso de inversiones para las instituciones.

Para la atención del fenómeno se hace necesario la intervención de toda la comunidad académica, pues debido a que la procrastinaciónes generada por situaciones endógenas y exógenas, es de suma importancia contar con todos actores del proceso educativo, con el fin de intervenir de manera oportuna y mitigar sus efectos en el trasegar académico.

\section{Agradecimientos}

A nuestras familias que siempre han estado apoyándonos en todos los retos que asumimos.

A nuestros docentes que han sido guía incondicional en este camino de adversidades, gracias por esos buenos consejos que nos ayudan a formarnos como excelentes profesionales.

A nuestra Universidad de la Amazonía por apoyarnos en nuestra formación.

A nuestra región Amazónica por brindarnos esa riqueza que inspira y fomentar el crecimiento.

\section{Referencias}

Arreola-Caro, M. (2012). "Evaluación holística del modelo pedagógico del Centro Universitario de los Valles de la Universidad de Guadalajara" (tesis doctoral). Recuperada en: http:/uvadoc.uva.es/handle/10324/1436

Barragán, S., Bogoya, D. \& Rodríguez, R. (2010) Hacia la retención estudiantil en la Universidad Jorge Tadeo Lozano. Universidad Jorge Tadeo Lozano. Disponible en: h t t p : / / w w w . ut adeo.edu.co/es/nuestraproduccion/departamento-de-ciencias basicas/5121/hacia-la-retencion-estudiantil-en-la

Buentello-Martínez, C.P., Valenzuela-Salazar, N.L. \& Juárez-Ibarra, D.P. (2014). Deserción escolar, factores que determinan el abandono de la carrera profesional estrategias y condiciones para el desarrollo del estudiante.Tesis doctoral. Recuperado en: http://www.fca.uach.mx/apcam/2014/04/04/Ponencia\%20 69-UACoah-Piedras\%20Negras.pdf

Caballero, J., Giacometty, L., Solano, 1., Gutierrez, J. \&Yusán, Y, (2012) Hacia La Construcción Colectiva De Un Marco Conceptual Para Analizar, Predecir, Evaluar Y Atender El Abandono Estudiantil En La Educación Superior (Guía, 2012). Universidad de Antioquia y Consejo Académico de la Universidad Santander.

Calvo, F. (2014) Universidad Nacional: Cambiar De Carrera O Programa Académico En Pregrado Y Postgrado. Universidad Nacional de Colombia.

Castañeda, R. (2013). Factores Asociados A La Deserción De Estudiantes Universitarios. (ICED - Tesis doctoral). Universidad de San Martin de Porres en LimaPerú.

Carbajal, S., (2012). La permanencia del estudiante durante el año de ingreso a la Universidad de la República. Una construcción colectiva. Inter Cambios,1(1),1-15. Recuperado de: http://intercambios.cse.edu.uy.

Carrión, D., Sanmartín, S., \& Fernández, M.S. (2012). "Facilitadores y obstáculos en las trayectorias de los 
estudiantes en la universidad nacional de general sarmiento" (tesis doctoral). Recuperado en: http:/iice.institutos.filo.uba.ar/sites/iice.institutos.filo.uba .ar/files/10-Niveles\%20educativos 00000000.pdf

Cuchacovich, I.A, (2011). "Retención y movilidad universitaria: Evaluación de Impacto de una Política" (tesis doctoral). Recuperada en: http://economia.uc.cl/wpcontent/uploads/2015/07/tesis_icuchacovich.pdf

De-Vries, W., León-Arenas, P., Romero-Muñoz, J.F \& Hernández-Saldaña, I. (2010). “¿Desertores o decepcionados? Distintas causas para abandonar los estudios universitarios". Revista de la Educación Superior, 40 ( 160 ) 1 - 16 . R e c u p e r a d o e n : http://www.scielo.org.mx/scielo.php?pid=S018527602011000400002\&script $=$ sci_arttext

Donoso, S., Donoso, G. \& Arias, O. (2010). “Iniciativas de retención de estudiantes en educación superior". Instituto de Educación y Desarrollo Educacional, 33(1)549 . R e c u p e r a d o e n : http://www.alfaguia.org/alfaguia/files/1319073270_04.pd $\mathrm{f}$

Dupéré, V., Leventhal, T., Dion, E., Crosnoe, R., Archambault, I., \&Janosz, M. (Diciembre 2015). Stressors and Turning Points in High School and Dropout A Stress Process, Life Course Framework. Review of Educational $\mathrm{R}$ e s e a r c h, 85 ( 4 ), $591-629$. D o i : $10.3102 / 0034654314559845$.

Escanés, G., Herrero, V., Merlino, A, \&Ayllon, S. (2013). "Aplicación de un modelo de duración en programas de prevención de deserción universitaria". Departamento de Investigación. Revista Electrónica de Investigación, 15(3)38-52. Recuperado en: https://dialnet.unirioja.es/servlet/articulo? codigo $=463889$ 9

Freeman, J., \&Simonsen, B. (Junio 2015). Examining the Impact of Policy and Practice Interventions on High School Dropout and School Completion Rates A Systematic Review of the Literature. Review of Educational Research, 85(2), 205-248. Doi: $10.3102 / 0034654314554431$.

Frelin, A. (Diciembre 2015). Relational underpinnings and professionality - a case study of a teacher's practices involving students with experiences of school failure. SchoolPsychology International,36(6) 589-604. Doi: 10.1177/0143034315607412.

Garcia, M. (2010). Diseño y validación de un modelo de evaluación por competencias en la universidad. (Tesis doctoral). Universidad Autónoma de Barcelona en la ciudad de Balleterra, España. Recuperado en: http://www.tdx.cat/bitstream/handle/10803/5065/mjgsp1d
e1.pdf;jsessionid=69B7D8233FB7DE5CC80E8731DE2C 2B80.tdx 1 ? sequence $=1$

García-deFanelli, A.M. (2014). "Rendimiento académico y abandono universitario: Modelos, resultados y alcances de la producción académica en la Argentina". Revista Argentina de Educación Superior, 6(8)9-38. Recuperado en: http://www.cedes.org.ar/67760CCD-

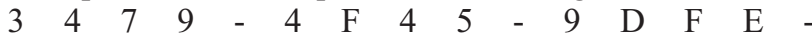
47 CCDC92180C/FinalDownload/DownloadId415F0CB4CEBF4A10965FDF363EBB8C6E/67760CCD - 3479 - 4 F 45 - 9 D F E 47CCDC92180C/PUBLICACIONES/EDSUP/2014/1064 6.pdf

González-López, I. (2010). “Motivación y actitudes del alumnado universitario al inicio de la carrera. ¿Varían al regresar?". Revista Electrónica de Investigación Psicoeducativa y Psicopedagógica, 5-3(1)35-56. Recuperado en: http://www.investigacionpsicopedagogica.org/revista/articulos/5/espannol/Art_5_2 9.pdf

Gómez C, CA., Tovar C, G. (2016). Factores endógenos causantes de la permanencia irregular en la Facultad de Ciencias Contables, Económicas y Administrativas de la Universidad de la Amazonia. Tesis de Maestría en Educación. Universidad de la Amazonia, Florencia, Caquetá(Colombia).

Gómez C, CA., Sánchez C, V., González, G. (2016). Reflexiones en torno a las políticas públicas. En: Revista FACCEA, volumen 6, numero 1. Facultad de Ciencias Contables, Económicas y Administrativas, Universidad de la Amazonia, Florencia, Caquetá (Colombia).

Hernández-Moreno, E.M. (2010). “Aprendizajes, competencias y rendimiento académico en la titulación de estudios socioculturales de la universidad de Cienfuegos" ( t e s i s doctoral). Re cuperad a e n : http://digibug.ugr.es/67760CCD-3479-4F45-9DFE$47 \mathrm{CCDC} 92180 \mathrm{C} / \mathrm{FinalDownload} / \mathrm{DownloadId-}$ 18C21B7EC0E0C5F0FBE3663D5A31751F/67760CCD-

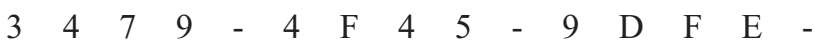
47CCDC92180C/bitstream/10481/4971/1/18709576.pdf

Lopera, J. \& Ortiz, E. (2012) Reflexiones en torno a las prácticas pedagógicas y didácticas de la lectura y la escritura en un programa de retención estudiantil universitaria. Universidad Tecnológica de Pereira. D i s p o n i b l e e n : http://www.utp.edu.co/vicerrectoria/investigaciones/publi caciones-lectura-escritura/referencia/ver/1306

Molina, N. \& Contreras, A. (2012). Los sistemas de acceso, normativas de permanencia, y estrategias de tutoría y retención de estudiantes de educación superior en Venezuela. Universidad de los Andes, Colombia. 
Disponible en:

http://accelera.uab.cat/ACCEDES/docs/informes/venezue la.pdf

Montes, I. \& Lerner, J. (2011). Rendimiento académico de los estudiantes de pregrado de la Universidad EAFIT. Colombia: Medellín. Recuperado de: http://www.eafit.edu.co/institucional/calidadeafit/investigacion/Documents/Rendimiento $\% 20 \mathrm{Ac} \% \mathrm{C} 3$ \%A1demico-Perrspectiva\%20cuantitativa.pdf

Mori, M. (2012). Deserción Universitaria En Estudiantes De Una Universidad Privada De Iquitos. Revista Digital de Investigación en Docencia Universitaria, 6(1) 60-83.

Natividad-Sáez, L. (2014). “Análisis de la procrastinación en estudiantes universitarios" (tesis d o c t o r a l). R e c u p e r a d a e n : http://www.academia.edu/8946570/UNIVERSITAT_DE_ VAL $\%$ C $3 \% 88$ NCIA

Hirschhorn, A. (2010). "Factores que facilitan y que dificultan la culminación de las tesis. Análisis comprobado de tres escuelas de postgrado en ciencias agropecuarias (argentina)" (tesis doctoral). Recuperado en: http://nulan.mdp.edu.ar/1625/1/hirschhorn_an.pdf

Olivete, S. (2010). "Estrés académico en estudiantes que cursan primer año del ámbito universitario" (tesis d o c t o r a l). R e c u p e r a d a d e : http://imgbiblio.vaneduc.edu.ar/67760CCD-3479-4F459DFE-47CCDC92180C/FinalDownload/DownloadId322B60BA9144EA5CF5AB1F6C16178300/67760CCD$\begin{array}{llllllllllllllll}3 & 4 & 7 & 9 & - & 4 & \mathrm{~F} & 4 & 5 & - & 9 & \mathrm{D} & \mathrm{F} & \mathrm{E} & -\end{array}$ 47CCDC92180C/fulltext/files/TC104100.pdf

Pey, R., Durán, F. \& Jorquera, P. (2013). "Informe para la toma de decisiones sobre Duración de las carreras de pregrado en el CRUCh" (tesis doctoral).Recuperado en: http://sct-chile.consejoderectores.cl/67760CCD-3479$\begin{array}{lllllllll}4 & \mathrm{~F} & 4 & 5 & - & 9 & \mathrm{D} & \mathrm{F} & \mathrm{E}\end{array}$ 47CCDC $92180 \mathrm{C} /$ FinalDownload/DownloadI4DC994EE2F92C57861FCB7D6748AF234/67760CCD$\begin{array}{lllllllllllllllll}3 & 4 & 7 & 9 & - & 4 & \mathrm{~F} & 4 & 5 & - & 9 & \mathrm{D} & \mathrm{F} & \mathrm{E} & -\end{array}$ 47CCDC92180C/documentos WEB/Innovacion Curricu lar/4.Duracion_de_las_carreras\%20de_pregrado_en_el_C RUCh.pdf

Parra, D. \&Rodríguez, L. (2014). Factores Que Inciden En La Permanencia Académica De Los Estudiantes De La Universidad Nacional Abierta Y A Distancia "Unad"- Cead Facatativá. Universidad Nacional Abierta y a Distancia UNAD.

Peralta, R. \& Mora, J. (2014) Diseño de estrategias para disminuir los índices de deserción de los beneficiarios de crédito educativo en tres países de América. Asociación Panamericana de Instituciones de Crédito Educativo.
Disponible en: http://www.alfaguia.org/wwwalfa/images/PonenciasClabes/1/ponencia_16.pdf

Pineda, C. (2013). Compromiso estudiantil y desempeño académico universitario: Comprobando el vínculo. (Tesis doctoral). Universidad de la Sabana, Chia, Colombia.

Pineda, C., Pedraza, A. \& Moreno, I. (2011). Efectividad de las estrategias de retención universitaria: la función del docente. Universidad de la Sabana-Chia, Cundinamarca, Colombia. Disponible en: http://educacionyeducadores.unisabana.edu.co/index.php/ eye/article/view/1833/2411

Prieto, D. \& Raleigh, R. (2013). "Propiedades psicométricas de una escala de procrastinación académica en estudiantes universitarios" (tesis doctoral). Recuperada en: http://200.35.84.131/portal/bases/marc/texto/3201-1305969.pdf

Proyecto ALFA (2013) "Encuesta Internacional sobre el Abandono en la Educación Superior: Hacia la construcción colectiva de un marco conceptual para analizar, predecir, evaluar y atender el abandono estudiantil en la educación superior" Guia Gestión Universitaria Integral del Abandono. Proyecto ALFA GUIA DCI-ALA/2010/94. Recuperado en: http://www.alfaguia.org/wwwalfa/images/resultados/Marco_Conceptual_sobre_elAbandono.pdf

Ramírez, H. y Vélez, R. (2010). La deserción académica y las estrategias gerenciales como punto de partida en las instituciones de educación superior. Universidad de Medellin, Colombia. Disponible en: http://repository.udem.edu.co/bits tream/handle/11407/56/ LA\%20DESERCI\%C3\%93N\%20ACAD $\%$ C3\%89MICA \%20Y\%20LAS\%20ESTRATEGIAS\%20GERENCIALE $\mathrm{S} \% 20 \mathrm{COMO}$.pdf? sequence $=1 \&$ isAllowed $=\mathrm{y}$

Regueyra-Edelman, M.G. (2014). “Aprendizajes sobre la población estudiantil que no continúa y el derecho a la educación superior" (tesis doctoral). Recuperado en: h t t p : / / w w w . a l f a g u i a. o r g/w w w alfa/images/ponencias/clabesIII/LT_1/ponencia_completa 8.pdf

Rizo-Bonilla, Y.A. (2010). "Factores que afectan las distintas formas de culminación de estudios que realizan los(as) estudiantes de la carrera administración de empresas, de la modalidad por encuentro dominical de la universidad de las américas (ulam) Managua". Revista Catedra, 1 (1 ) 1-127. R e cuperado e n: http://revistacatedra.unan.edu.ni/index.php/investigacione s/article/view/334/304

Rodríguez-Pérez, J., Morales-Macías, S., \&RodriguezMorales, J. (2012). "Determinación de las causas por las cuales los alumnos no se titulan" (tesis doctoral). 
Recuperada en:

http://www.apcam.org.mx/Documentos/PONENCIA $\% 20$ 142-UACH.pdf

Sánchez, A. (2010). Procrastinación académica: un problema en la vida universitaria.Revista FuncaciónDialnet, Studiositas, ISSN-e 1909-0366, Vol. 5, $\mathrm{N}^{0}$. 2, 2010, págs. 87-94. Recuperado de: https://dialnet.unirioja.es/servlet/articulo? codigo $=371732$ 1

Shefi, Y. (Diciembre 2015). The Contribution of Teacher-Student Relationships to Perseverance, Dropout Prevention and Motivation for Change in Students' Attitudes in "Second Chance" High School. ProcediaSocial and Behavior Sciences. 209(2015)470-475. Doi: 10.1016/j.sbspro.2015.11.255.

Suarez, N. \&Díaz, L. (2015). "Estrés académico, deserción y estrategias de retención de estudiantes en la educación superior". Rev. Salud Pública,17(2),300-313. $\mathrm{R}$ e c u p e r a d o e $n$ http://www.scielo.org.co/pdf/rsap/v17n2/v17n2a13.pdf

Tonconi-Quispe, J. (2010). "Factores Que Influyen En El Rendimiento Académico Y La Deserción De Los Estudiantes De La Facultad De Ingeniería Económica De La Una-Puno, Periodo 2009". Cuadernos de Educación y Desarrollo, 2( 11 ) 1-22. Recuperado en: http://www.eumed.net/rev/ced/11/jtq.htm

Torres, L. (2012). Retención estudiantil en la educación superior: revisión de la literatura y elementos de un modelo para el contexto colombiano. Colombia: Bogotá.

Torres, L., (Julio de 2010). Estado del arte de la retención de estudiantes de la educación superior. Pontificia Universidad Javeriana Facultad De Educación Secretaría De Planeación Bogotá, Colombia. Disponible en: https://contextoseducativosinteractivos.files.wordpress.co m/2012/11/estado_del_arte_de_la_retencion_de_estudian tes.pdf

Ulloa-Garrido, J., Gajardo-Aguayo, J, \& DíazChinchilla, M. (2015). "Percepciones sobre la trayectoria socio-académica de estudiantes participantes del Programa Propedéutico de la Universidad de Concepción". Revista de Investigación Educacional Latinoamericana, 52(1)33-46. Recuperado en:

http://pensamientoeducativo.uc.cl/files/journals/2/articles/ 685/public/685-2151-1-PB.pdf
Universidad Distrital Francisco José de Caldas. (2011). Retención de Estudiantes.Oficina Asesora de Planeación y C o n t r o l. $\quad$ R e c u p e r a d o d e http://comunidad.udistrital.edu.co/planeacion/files/201 1/1 0/Estudio-de-Retenci\%C3\%B3n-en-la-UD.pdf

Trejo, A.P. \& Sierra-Paycha, C. (2014). “Gestión de la movilidad estudiantil en Estados Unidos. Dimensionando a los estudiantes colombianos y mexicanos". Camino Real, 6 ( 9 ) $119-141$. R e c u p e r a d o e n : http:/www.institutofranklin.net/67760CCD-3479-4F459DFE-47CCDC92180C/FinalDownload/DownloadId7295137FE21D376B5E41081EDDCD0D78/67760CCD-

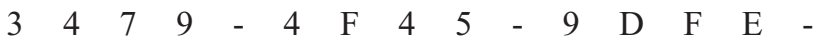
47CCDC92180C/sites/default/files/files/TREJO\%20Y\%2 0SIERRA.pdf

Vallejos, M. (2012). La Motivación, La Actitud Hacia Las Ciencias, La Ansiedad Y Las Estrategias Metacognitivas De Lectura En El Rendimiento De Los Estudiantes Universitarios: Un Análisis Longitudinal. (Tesis doctoral). Universidad Complutense de Madrid, España. Recuperado en: http://eprints.ucm.es/17020/

Villa, E., Misas, M., Berrio, M., \& Santacruz, S. (Noviembre de 2013) Un Modelo de Educación Superior y Deserción Universitaria: Evidencia de la Pontificia Universidad Javeriana-Bogotá. Universidad Javeriana B o g o t á. $\quad$ D i s p o n i b l e e n : http://cea.javeriana.edu.co/documents/153049/2786252/V ol.13_8_2013.pdf/26235e24-083b-44f6-8b57504ea $\overline{8} 28 \overline{\mathrm{d}}$ ee 6

Zarate, R. \&Socha, C. (2010). Estudio sobre las motivaciones de deserción estudiantil en la Universidad Industrial de Santander. Universidad Industrial de Santander-Bucaramanga, Colombia. Disponible en: http://www.colombiaaprende.edu.co/html/micrositios/175 2/articles-345093_recurso_3.pdf 